\title{
Greater osteoblast proliferation on anodized nanotubular titanium upon electrical stimulation
}

\author{
Batur Ercan' \\ Thomas J Webster ${ }^{1,2}$ \\ 'Division of Engineering, Brown \\ University, Providence, RI, USA; \\ ${ }^{2}$ Department of Orthopedic Surgery, \\ Brown University, Providence, RI, USA
}

Correspondence:Thomas JWebster Division of Engineering and Department of Orthopedics, Brown University, I82 Hope Street, Box D, Providence, RI, USA 02912

Tel + I 4018632318

Fax +14018639107

Email thomas_webster@brown.edu

\begin{abstract}
Currently used orthopedic implants composed of titanium have a limited functional lifetime of only 10-15 years. One of the reasons for this persistent problem is the poor prolonged ability of titanium to remain bonded to juxtaposed bone. It has been proposed to modify titanium through anodization to create a novel nanotubular topography in order to improve cytocompatibility properties necessary for the prolonged attachment of orthopedic implants to surrounding bone. Additionally, electrical stimulation has been used in orthopedics to heal bone non-unions and fractures in anatomically difficult to operate sites (such as the spine). In this study, these two approaches were combined as the efficacy of electrical stimulation to promote osteoblast (bone forming cell) density on anodized titanium was investigated. To do this, osteoblast proliferation experiments lasting up to 5 days were conducted as cells were stimulated with constant bipolar pulses at a frequency of $20 \mathrm{~Hz}$ and a pulse duration of $0.4 \mathrm{~ms}$ each day for 1 hour. The stimulation voltages were $1 \mathrm{~V}, 5 \mathrm{~V}, 10 \mathrm{~V}$, and $15 \mathrm{~V}$. Results showed for the first time that under electrical stimulation, osteoblast proliferation on anodized titanium was enhanced at lower voltages compared to what was observed on conventional (nonanodized) titanium. In addition, compared to nonstimulated conventional titanium, osteoblast proliferation was enhanced $72 \%$ after 5 days of culture on anodized nanotubular titanium at $15 \mathrm{~V}$ of electrical stimulation. Thus, results of this study suggest that coupling the positive influences of electrical stimulation and nanotubular features on anodized titanium may improve osteoblast responses necessary for enhanced orthopedic implant efficacy.
\end{abstract}

Keywords: titanium, anodization, nanotubular, electrical stimulation, osteoblast, proliferation

\section{Introduction}

Titanium is one of the most commonly used implant materials in orthopedics. Although titanium has excellent corrosion resistance and suitable mechanical properties to support physiological loads, its cytocompatibility properties are not sufficient to maintain the implant functionality necessary to heal bone fractures over long periods of time. In fact, conventional titanium-based implants only have functional lifetimes of 10-15 years, making it necessary for younger patients to have at least one revision surgery, and in some cases multiple revision surgeries, before the end of their lives. Specifically, the long-term failure of currently used titanium implants is due to clinical problems such as extensive prolonged fibrous tissue encapsulation, wear debris, infection, stress shielding, etc. Clearly, currently used titanium-based implants fail to satisfy the needs of all patients and improvements are necessary.

The use of nano-structured materials has been proposed to solve some of the aforementioned problems currently associated with orthopedic implants. Nano-structured materials are those materials with at least one dimension less than $100 \mathrm{~nm}$. The main reason for exploring nano-structured materials in orthopedics is that bone itself is a nano-structured tissue. For instance, hydroxyapatite crystals, the main constituent of the inorganic phase of bone, are 2-5 nm thick and collagen type I fibrils, the main constituent of the organic phase of bone, are $0.5 \mathrm{~nm}$ in diameter. This implies that bone 
cells are naturally accustomed to interacting with nanoscale surface features in the body and even synthesize such nanostructured materials.

It has been speculated that when implant surfaces are engineered to mimic the dimensions of the constituent components of bone, a better integration of the implant to surrounding bone can be expected. This is because compared to conventional (or nano-smooth materials) nano-rough materials have more surface area, surface defects, increased numbers of atoms, and altered electron distributions. Collectively, such properties inherent for nano-structured materials change surface reactivity with proteins and subsequently cells compared to conventional materials. Indeed, experimentally, changes in these surface properties on titanium (through anodization which creates novel nanotubular structures) influence the concentration and conformation of adsorbed proteins to alter cellular interactions.

Specifically, Yao and colleagues (2005) observed a $33 \%$ increase in osteoblast adhesion on anodized titanium surfaces with respect to conventional titanium. The increase in osteoblast adhesion was correlated with an $18 \%$ increase in vitronectin adsorption and a 30\% increase in fibronectin adsorption on anodized compared to conventional titanium (Yao et al 2005). Additionally, osteoblasts were more well spread and they were shown to deposit more calciumcontaining mineral on anodized nanotubular titanium (a crucial step for the regeneration of bone) compared to conventional titanium. Rodriguez and colleagues (2002) observed greater osteocalcin production by osteoblasts cultured on anodized compared to unanodized titanium surfaces (Rodriguez et al 2002). Lastly, animal experiments recently confirmed a higher interfacial strength between anodized titanium and juxtaposed tissue (Son et al 2003).

Modifying the surfaces of titanium to possess novel nanotubular structures is not the only way to promote bone bonding. The building blocks of living organisms, ions, polar/charged molecules, etc., all create and respond to electrical fields (Kirson et al 2007). For instance, bone has strain and strain rate dependant forward and reverse polarizations that create 10-20 $\mu \mathrm{A}$ currents (Black 1987). In their natural niche, many tissues are exposed to different levels of currents and electrical fields. In fact, electrical stimulation has been used in a number of clinical applications in orthopedics. For example, the proposed methodologies for using electrical stimulation for orthopedic applications varies from anterior and posterior placement of electrodes around a hip implant, to coiling them around with a subcutaneous power source, all the way to placement of an electromagnetic coil at the surgical bed-side. In fact, electrical stimulation has been used clinically for some patients undergoing spinal fusion surgeries, nonunion fractures, and for prior failed joint fusions who suffer from poor bone growth or diseases which cause bone to regrow slowly (Hartig et al 2000; Nelson et al 2003; Aaron et al 2004a).

Some have already combined the benefits of nano-structured materials and electrical stimulation for orthopedic applications and have found exciting results. For example, for in vitro experiments, carbon nanotubes (CNT) were added to poly-lactic acid (PLA) (Supronowicz et al 2001). Although PLA is an insulative material, $20 \mathrm{wt} . \%$ addition of CNT transformed PLA into a conductive material. When this composite was exposed to $10 \mu \mathrm{A}$ of an alternating current for 6 hours a day, a 46\% increase in osteoblast proliferation was observed after 2 days and a 307\% increase in calcium deposition by osteoblasts was observed after 21 days with respect to nonstimulated samples (Supronowicz et al 2001). Similar results were also observed by Hartig and colleagues (2000) in which the application of a capacitively coupled electrical field increased primary osteoblast-like cell proliferation, alkaline phosphatase activity and extracellular matrix protein synthesis (Hartig et al 2000; Aaron et al 2004a). Khang and colleagues (2008) also combined nano-structured materials and electrical stimulation for cartilage applications by demonstrating enhanced proliferation of chondrocytes (cartilage synthesizing cells) on electrically stimulated $\mathrm{CNT} /$ poly-carbonate urethane composites compared to nonstimulated respective substrates.

However, to the best of our knowledge, there has not been any research concerning combining electrical stimulation with one of the most commonly implanted orthopedic biomaterials, titanium, modified to have nano-structured surface features. Due to this reason, the current in vitro study investigated the proliferation of osteoblasts on anodized nanotubular titanium under 4 different voltages for up to 5 days of culture.

\section{Experimental procedures Specimen preparation}

99.2\% pure titanium foils (1.6 mm thick) were purchased (Alfa Aesar, Ward Hill, MA) and cut into $1 \mathrm{~cm} \times 1 \mathrm{~cm}$ squares. Then, each side of these squares were sonicated (VWR 75D) for 1 hour using a cleaning solution (Branson, IS), followed by 30 minutes of sonication with acetone (Mallinckrodt Chemicals), 70\% ethanol and $\mathrm{ddH}_{2} \mathrm{O}$. These cleaned samples were termed 'conventional titanium' in the present study. 
Afterwards, the surface cleaned titanium samples were etched with a $1.5 \% \mathrm{HF} / 1.5 \% \mathrm{HNO}_{3}$ solution (Mallinckrodt Chemicals) for 5 minutes to remove the oxide layer. This was followed by an anodization process (Figure 1). For anodization, titanium samples were connected to a DC-powered electrochemical cell which had a two electrode configuration. A platinum mesh was used as the cathode and a titanium specimen was used as the anode. These platinum and titanium samples were connected to a DC power supply (3645A DC power supply, Circuit Specialists, Inc.) through copper wires. A constant voltage of $20 \mathrm{~V}$ was applied for 10 minutes according to previous studies (Yao et al 2005). The distance between the titanium anode and platinum cathode was kept constant at $1 \mathrm{~cm}$. The electrolyte solution used in this study was $1.5 \%$ hydrofluoric acid and the anodization was conducted inside a Teflon beaker. During anodization, the electrolyte solution was constantly stirred with magnetic agitation to reduce the thickness of the double layer at the metal-electrode interface to obtain uniform local current densities on the titanium electrode (Mor and Varghese 2003). After anodization, the specimens were again sonicated with acetone, $70 \%$ ethanol and $\mathrm{ddH}_{2} \mathrm{O}$, for 30 minutes each. These samples were termed 'anodized nanotubular titanium' since, as will be shown, titanium anodized in this manner possesses nanotubes penetrating the titanium surfaces.

\section{Material characterization}

The surfaces were characterized using a LEO 1530 VP FE-4800 Field-Emission Scanning Electron Microscope (SEM). $5 \mathrm{kV}$ accelerating voltages were used to image the specimens. No coatings were used to image the surfaces.

\section{Electrical stimulation}

A multi channel electrical stimulator (Ionoptix, Milton, MA) was used to stimulate osteoblasts with electricity. This stimulator generates bipolar pulses at a frequency of

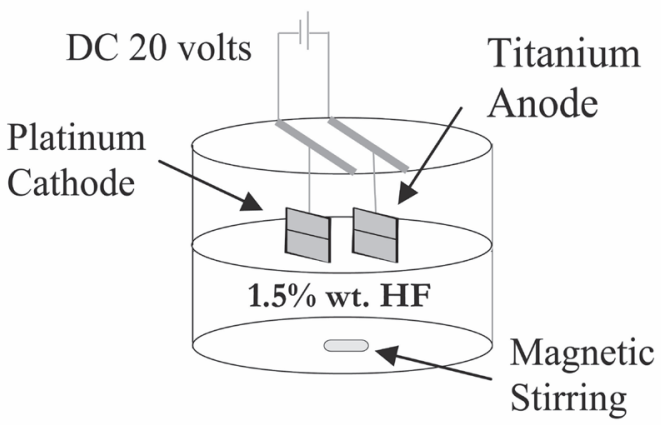

Figure I Schematic of the anodization system.
0.01 to $99 \mathrm{~Hz}$, pulse duration of $0.4-24 \mathrm{msec}$, and voltages of up to $\pm 40 \mathrm{~V}$. There was net charge transport between the electrodes of the electrical stimulation system and the primary field created was an electrical field as opposed to a magnetic field. The stimulator had two components, a voltage generator and a cell culture dish electrode assembly (Figure 2). A voltage generator was connected to the culture dish electrode assembly through a thin ribbon cable, which allowed for a good seal with the cell culture incubator door. Each dish was stimulated separately with a slight time delay, on the order of milliseconds. The resistance (R) of each well was calculated from equation 1 :

$$
R=\frac{d}{\sigma * \mathrm{~A}}
$$

where d stands for distance between graphite electrodes, A stands for the area of the electrodes in the media, and $\sigma$ stands for the conductivity of the media. The distance between graphite electrodes was $3.2 \mathrm{~cm}$, the surface area of the electrode in contact with the media was $0.473 \mathrm{~cm}^{2}$ and the conductivity of the media at $36{ }^{\circ} \mathrm{C}$ was $0.0146 \mathrm{~S} / \mathrm{cm}$, giving a resistance of $463 \mathrm{Ohms}$ for each well. Upon the application of $1 \mathrm{~V}$ to the media, $2.16 \mathrm{~mA}$ passed through the media. However, the physiological current bone experiences is $10-20 \mu \mathrm{A}$, nearly 1000 times lower (Black 1987). In fact, during trial runs, $2.16 \mathrm{~mA}$ was found to be lethal for osteoblasts in the present study (data not shown). Therefore, a $75 \mathrm{k} \Omega$ potentiometer was connected to the circuit to mimic the physiological current osteoblasts are exposed to during normal activity. Upon the application of $1 \mathrm{~V}$ to the system, the current passing through the media was estimated to be $13 \mu \mathrm{A}$.

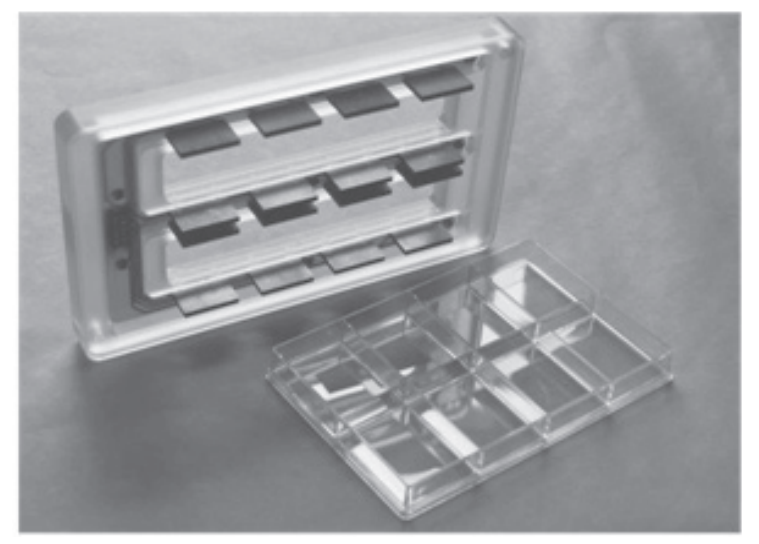

Figure 2 Schematic of the 8-well culture dish electrode assembly showing a uniquely manufactured upper lid with graphite electrodes. The electrodes were placed inside the media during electrical stimulation of the cells and the 8-well dish was connected to the banks of the voltage generator. 


\section{Cell culture}

Osteoblasts (population numbers 8 to 15 , American Type Culture Collection CEL-11372) were cultured in osteoblast cell culture medium (Dulbecco's Modified Eagle Medium [DMEM]; Hyclone, Logan, UT) supplemented with $10 \%$ fetal bovine serum (FBS; Hyclone) and 1\% penicillinstreptomycin $(\mathrm{P} / \mathrm{S}$; Hyclone) under standard culture conditions $\left(5 \% \mathrm{CO}_{2} / 95 \%\right.$ air at $\left.37{ }^{\circ} \mathrm{C}\right)$.

For the cell experiments, all samples were sterilized using a steam autoclave (Amsco Renascence Series, 3021). The osteoblasts in DMEM supplemented with $10 \%$ FBS and $1 \% \mathrm{P} / \mathrm{S}$ were seeded onto the specimens at a density of $3500 \mathrm{cell} / \mathrm{cm}^{2}$. Cells were cultured in the same media under standard cell culture conditions for 5 days and cell counts were performed at the $1 \mathrm{st}, 3 \mathrm{rd}$, and 5 th days. The media was changed at the 2 nd and 4 th days. At the end of the prescribed time period, specimens were gently rinsed in a $1 \times$ PBS buffer solution to remove nonadherent cells. Adherent cells were then fixed with $4 \%$ formaldehyde (Fisher Scientific, Pittsburgh, PA) for $10 \mathrm{~min}$ and nuclei stained by soaking in a $1 \%$ DAPI fluorescent dye (Sigma, St. Louis, MO) for $20 \mathrm{~min}$. Cell densities were counted in situ from five randomly selected, nonintersecting areas on each specimen under a fluorescence microscope (Leica DM 5500B). Numerical data was analyzed using standard analysis of variance (ANOVA). All cell experiments were repeated three times both for conventional and anodized nanotubular titanium samples.

\section{Cell experiments with electrical stimulation}

The first electrical stimulation was conducted $8 \mathrm{~h}$ after the cells were seeded onto the specimens. The duration of the electrical stimulation was $1 \mathrm{~h}$ and the cells were stimulated each day. For all experiments, the pulse duration and frequency were selected based on prior research and kept constant at $0.4 \mathrm{~ms}$ and $20 \mathrm{~Hz}$, respectively (Black 1987). The voltages used for these experiments were $1,5,10$, and $15 \mathrm{~V}$, corresponding to $13,65,130$, and $195 \mu \mathrm{A}$, respectively. No change was observed in $\mathrm{pH}$ or temperature of the media after $1 \mathrm{~h}$ of electrical stimulation.

\section{Results and discussion Material characterization}

As determined by FESEM, the surfaces of the conventional (unanodized) and anodized nanotubular titanium confirmed that observed in previous studies (Figure 3; Yao et al 2005).
Specifically, the anodization process created titania nanotubes on the titanium specimens. The diameters of the titania nanotubes ranged from $40-60 \mathrm{~nm}$. Different researchers have measured different nanotube depths, varying between $200-500 \mathrm{~nm}$ depending on the electrolyte concentrations, times, and titanium alloy compositions used (Mor and Varghese 2003; Lee et al 2006). The depths of the nanotubes created in the present study have been estimated at 200-250 nm (Yao et al 2005).

\section{Osteoblast cell number results}

After 1 day of culture, results of the present study confirmed those of other studies that have been completed without electrical stimulation. Specifically, without electrical stimulation, more osteoblasts were counted on anodized nanotubular compared to unanodized titanium after 1 day (Figure 4). Yao and colleagues (2005) first reported higher osteoblast density on anodized nanotubular compared to unanodized titanium after a 4-h adhesion test. Yao and colleagues (2005) continued to report significantly greater alkaline phosphate activity and calcium deposition by osteoblasts cultured on anodized nanotubular compared to unanodized titanium for up to 21 days.

Interestingly, Karlsson and colleagues (2003) reported similar greater osteoblast adhesion (when not electrically stimulated) on anodized compared to unanodized alumina. The fact that two different metals can be oxidized and promote osteoblast functions leads one to question whether any metal may fit this trend after anodization. Compared to unanodized titanium, Yao and colleagues (2005) explained the observed greater osteoblast adhesion on anodized nanotubular titanium by measuring increased adsorption of vitronectin and fibronectin onto the anodized titanium surfaces.

However, in addition to confirming the results of other studies (Yao et al 2005), the present study also provided the first evidence that osteoblasts responded to lower amounts of electrical stimulation when cultured on anodized nanotubular than unanodized titanium (Figure 4). Specifically, after 1 day of culture, statistically greater numbers of osteoblasts were counted on anodized nanotubular titanium electrically stimulated at $10 \mathrm{~V}$ compared to unstimulated anodized materials while the same event did not happen on unanodized titanium until $15 \mathrm{~V}$. After 3 days of culture, statistically greater numbers of osteoblasts were counted on anodized nanotubular titanium stimulated at $10 \mathrm{~V}$ compared to $5 \mathrm{~V}$; the same comparison was not statistically different until osteoblasts were stimulated at $15 \mathrm{~V}$ on conventional 
a)

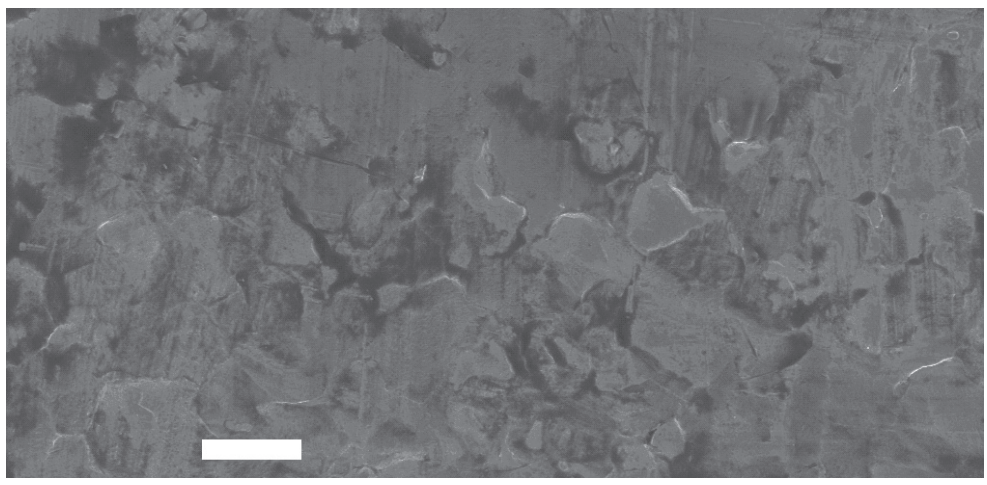

b)

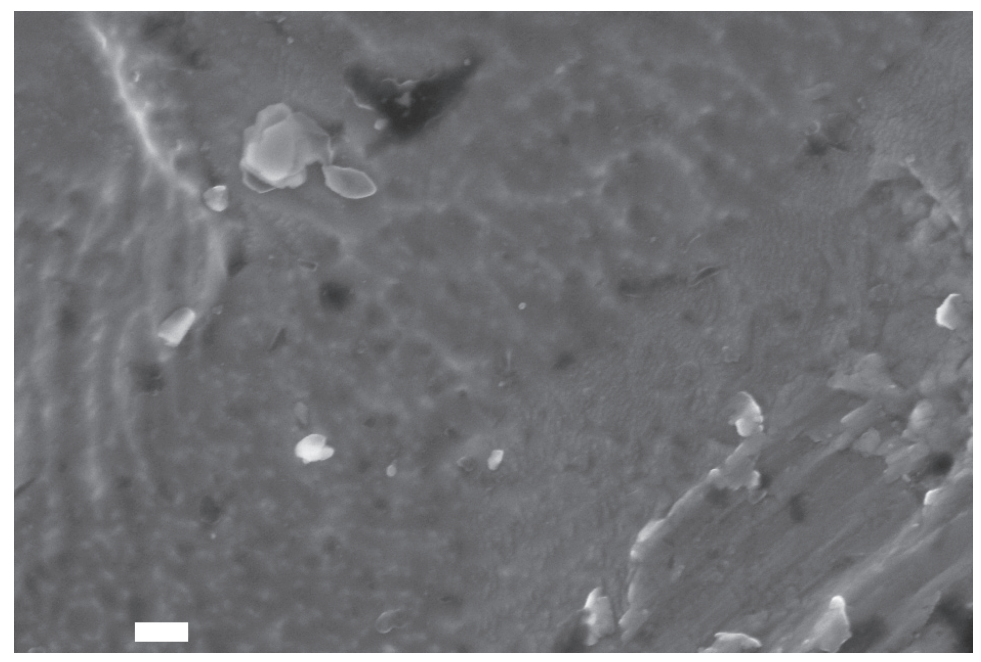

c)

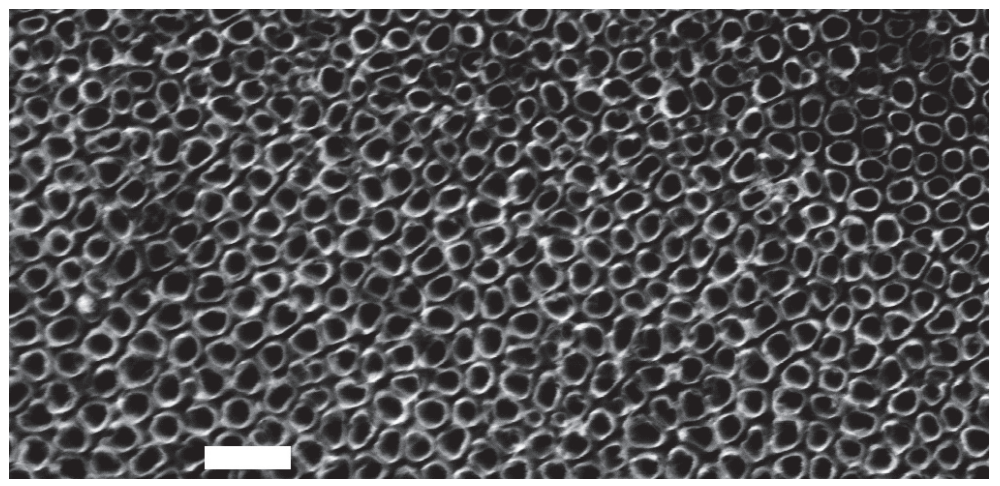

Figure 3 SEM images showing the surface features of: (a) (low magnification), (b) (high magnification) conventional, and (c) anodized nanotubular titanium. Notes: Scale bars are: (a, b) $10 \mu \mathrm{m}$, and (c) $200 \mathrm{~nm}$.

titanium (Figure 5). This same trend of less voltage increasing osteoblast densities on anodized nanotubular compared to unanodized titanium continued after 5 days of culture (Figure 6). Specifically, compared to unstimulated anodized materials, more osteoblasts were counted on anodized nanotubular titanium stimulated at $10 \mathrm{~V}$ while the same levels of osteoblast numbers were not observed until $15 \mathrm{~V}$ was reached on conventional, nonanodized titanium after 5 days of culture. Moreover, of any of the substrates of interest to the present study, the largest numbers of osteoblasts were counted on anodized nanotubular titanium electrically stimulated at $15 \mathrm{~V}$ after 5 days of culture (Figure 6).

As expected, the results of the present study also demonstrated that osteoblasts grew on both conventional and anodized nanotubular titanium with culture time (from 1 to 3 to 5 days) (Figures 7 and 8). Table 1 lists the percent increases in osteoblast numbers up to 5 days of culture on the substrates of interest to the present study. When the percent 


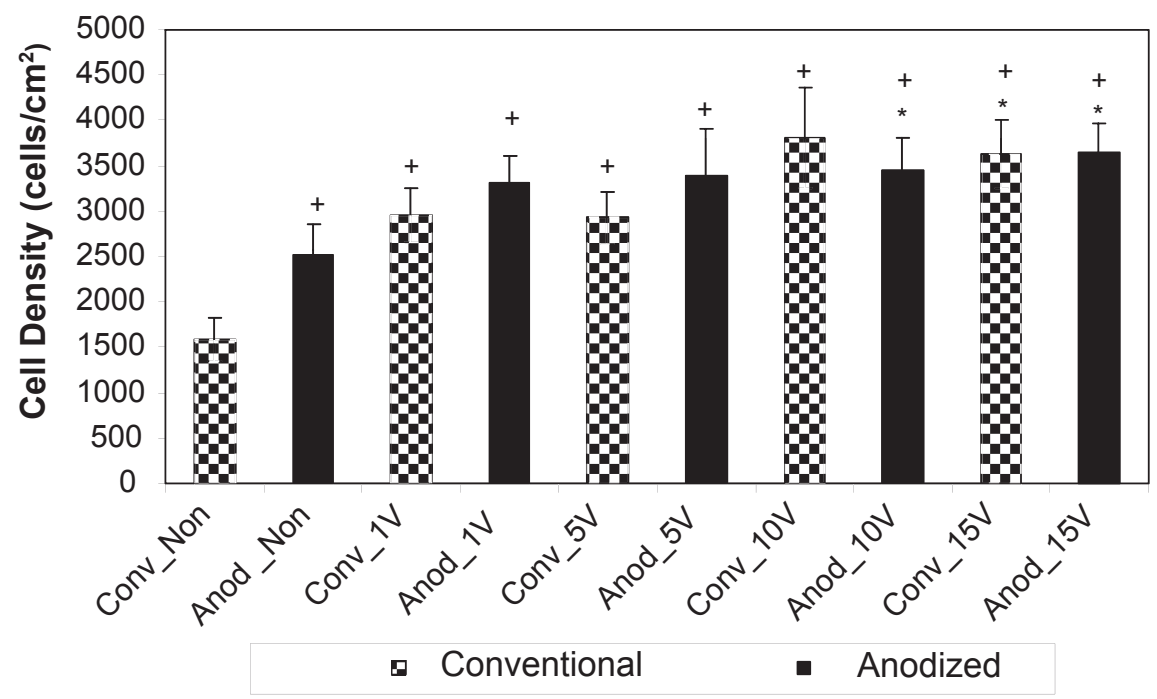

Figure 4 Osteoblast densities after I day on the nonstimulated as well as I V, $5 \mathrm{~V}, 10 \mathrm{~V}$, and I5V electrically stimulated conventional and anodized nanotubular titanium. Notes: Values are mean $\pm \mathrm{SEM} ; \mathrm{n}=3$; ${ }^{*} \mathrm{p}<0.05$ compared to Anod_Non; ${ }^{+} \mathrm{p}<0.05$ compared to Conv_Non.

Abbreviations: Anod, anodized nanotubular titanium; Conv, conventional (nonanodized titanium); Non, nonelectrical stimulated.

increases in osteoblast densities from the 1 st to 3 rd days of culture are compared, they were found to be higher for conventional titanium compared to anodized nanotubular titanium for all test conditions (excluding the $10 \mathrm{~V}$ stimulation, the same voltage consistently highlighted on anodized nanotubular titanium as the one in which osteoblasts performed statistically similar to osteoblasts on conventional titanium at $15 \mathrm{~V}$ ). Upon comparison of the percent increases in osteoblast densities from the 3rd to 5th days of culture, they were higher for osteoblasts on anodized compared to their conventional counterparts for all voltage conditions. In fact, when percent increases in cell densities from the 1 st to 3 rd days and from the 3rd to 5th days are compared, osteoblast proliferation was found to decrease with time more on conventional titanium samples than anodized nanotubular titanium. While the reasons for these trends are not

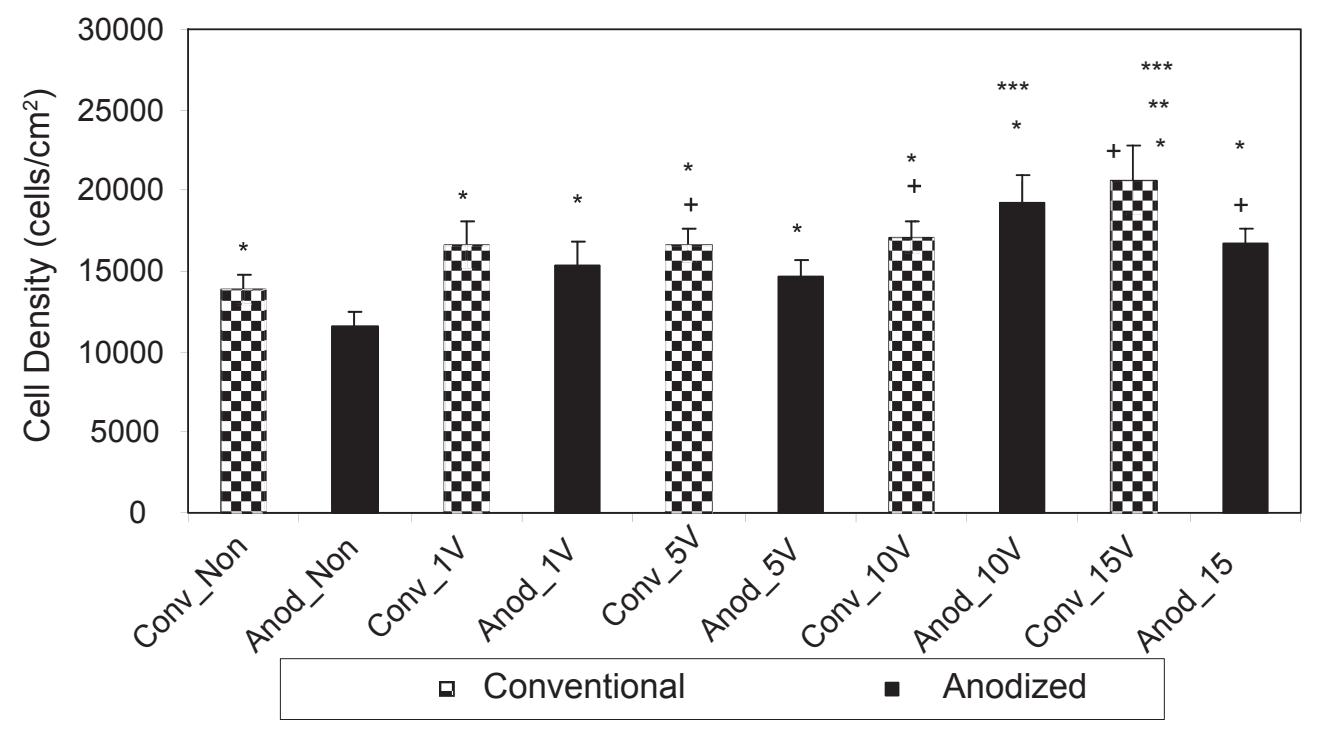

Figure 5 Osteoblast densities after 3 days on nonstimulated as well as I V, 5V, I0 V, and I5V electrically stimulated conventional and anodized nanotubular titanium. Notes: Values are mean $\pm \mathrm{SEM} ; \mathrm{n}=3{ }^{*}{ }^{\mathrm{p}} \mathrm{p}<0.05$ compared to Anod_Non; ${ }^{* *} \mathrm{p}<0.05$ compared to Anod_I V; ${ }^{* * *} \mathrm{p}<0.05$ compared to Anod_5 V; ${ }^{+} \mathrm{p}<0.05$ compared to Conv_Non.

Abbreviations: Anod, anodized nanotubular titanium; Conv, conventional (nonanodized titanium); Non, nonelectrical stimulated. 


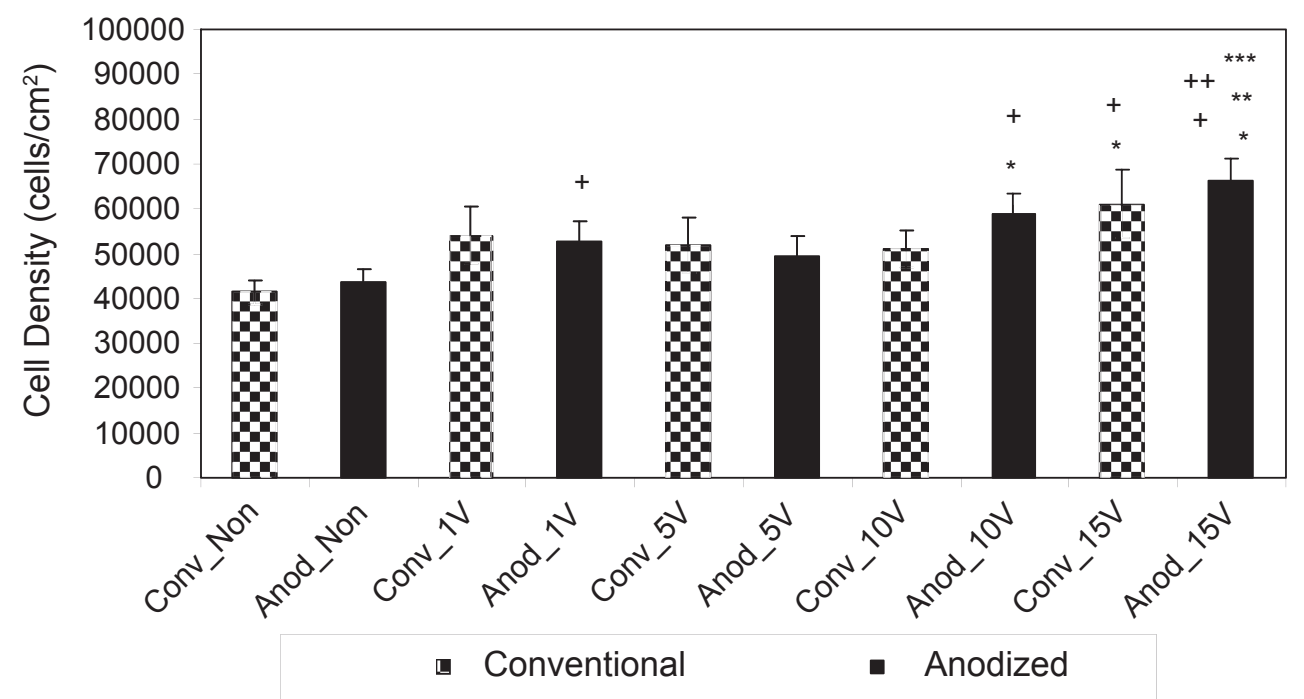

Figure 6 Osteoblast densities after 5 days on nonstimulated as well as IV, $5 \mathrm{~V}, 10 \mathrm{~V}$, and $15 \mathrm{~V}$ stimulated conventional and anodized nanotubular titanium specimens. Notes: Values are mean $\pm \mathrm{SEM} ; \mathrm{n}=3,{ }^{*} \mathrm{p}<0.05$ compared to Anod_Non; ${ }^{* *} \mathrm{p}<0.05$ compared to Anod_I V; ${ }^{* * *} \mathrm{p}<0.05$ compared to Anod_5 $\mathrm{V}$; ${ }^{+} \mathrm{P}<0.05$ compared to Conv_Non; ${ }^{++} \mathrm{p}<0.05$ compared to Conv_IOV.

Abbreviations: Anod, anodized nanotubular titanium; Conv, conventional (nonanodized titanium); Non, nonelectrical stimulated.

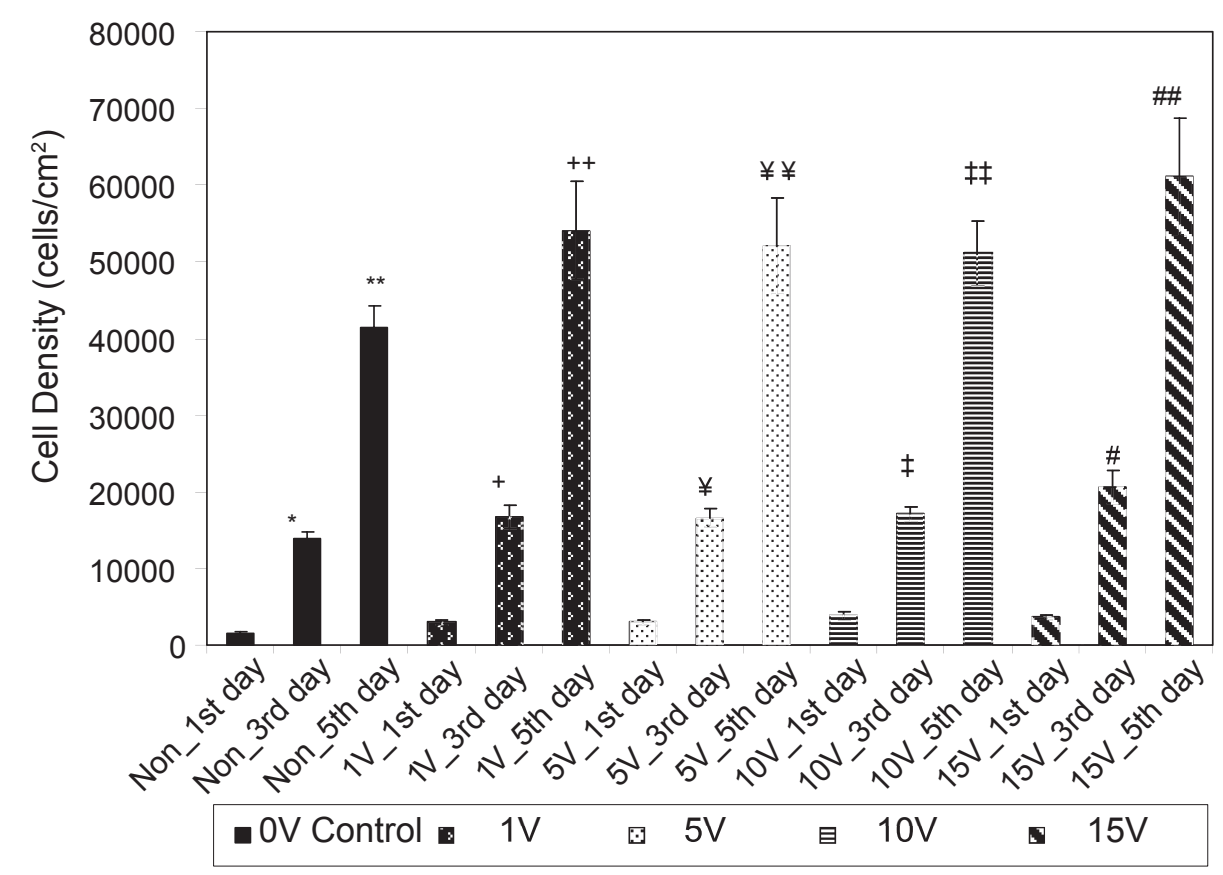

Figure 7 Osteoblast densities from I to 5 days on conventional (nonanodized) titanium.

Notes: Values are mean \pm SEM; $n=3 ;{ }^{*} p<0.05$ compared to Non_Ist day; ${ }^{* *} p<0.05$ compared to Non_3rd day; ${ }^{+} \mathrm{p}<0.05$ compared to I V_Ist day; ${ }^{++} \mathrm{p}<0.05$ compared to I V_3rd day; ${ }^{\ddagger} \mathrm{p}<0.05$ compared to $5 \mathrm{~V} \_$I st day; ${ }^{\ddagger} \mathrm{p}<0.05$ compared to $5 \mathrm{~V} \_3$ rd day; ${ }^{\ddagger} \mathrm{p}<0.05$ compared to $10 \mathrm{~V} \_$I st day; ${ }^{\ddagger \ddagger} \mathrm{P}<0.05$ compared to $10 \mathrm{~V} \_3$ rd day; $\#$ P $<0.05$ compared to $15 \mathrm{~V} \_$Ist day; and \# $\mathrm{p}<0.05$ compared to I5 V_3rd day. Abbreviation: Non, nonelectrical stimulated. 


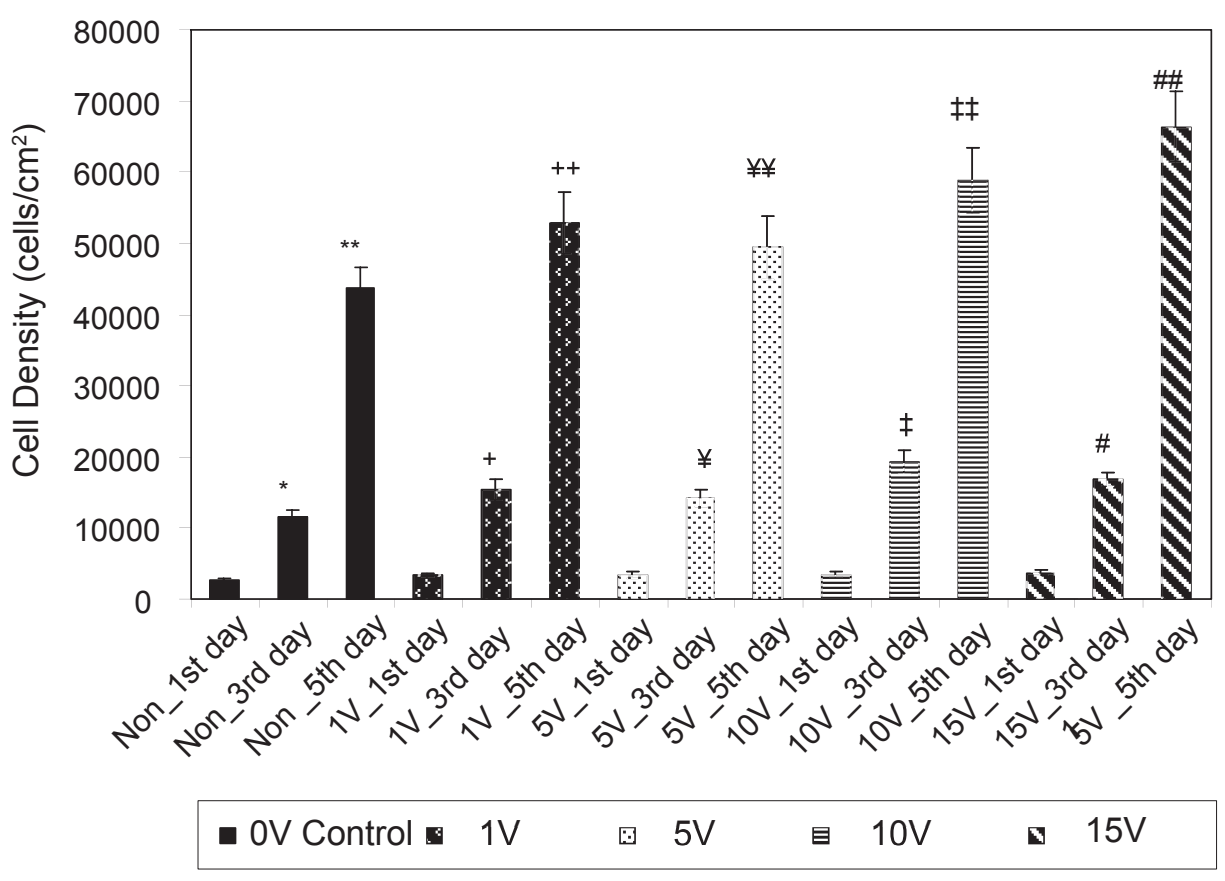

Figure 8 Osteoblast densities from I to 5 days on anodized nanotubular titanium.

Notes: Values are mean \pm SEM; $\mathrm{n}=3 ;{ }^{*} \mathrm{p}<0.05$ compared to Non_Ist day; ${ }^{* *} \mathrm{p}<0.05$ compared to Non_3rd day; ${ }^{+} \mathrm{p}<0.05$ compared to I $\mathrm{V} \_$Ist day; ${ }^{++} \mathrm{p}<0.05$

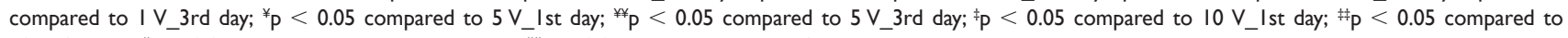
$10 \mathrm{~V} \_3$ rd day; ${ }^{\#} \mathrm{p}<0.05$ compared to $15 \mathrm{~V} \_$Ist day; and ${ }^{\mathrm{p}}<0.05$ compared to I5 V_3rd day.

Abbreviation: Non, nonelectrical stimulated.

clear at this time, it is important to note that, as mentioned, more osteoblasts were present on anodized nanotubular than conventional titanium after 1 day and it is possible that the combined effects of altered titanium surface properties obtained through anodization and electrical stimulation are responsible for these complex trends.

Additionally, from these results, one can conclude that electrical stimulation can successfully be used to increase osteoblast proliferation on anodized nanotubular titanium. The data show that the best osteoblast proliferation results were obtained by stimulating with either $10 \mathrm{~V}$ or $15 \mathrm{~V}$ for each investigated time point regardless of whether titanium was anodized. This indicates for the first time that within the tested voltage range, holding the frequency and pulse duration constant at $20 \mathrm{~Hz}$ and $0.4 \mathrm{~ms}$, the optimal voltage window for maximizing osteoblast densities on anodized titanium is $10 \mathrm{~V}$ to $15 \mathrm{~V}$. Most importantly, it was shown here that osteoblasts responded to lower voltages on anodized titanium compared to conventional titanium, perhaps because more cells initially adhered to anodized titanium, which would promote more focal contacts and cell-cell contacts allowing for less voltage to electrically stimulate cell growth.

Lastly, although it is still not completely understood, researchers have tried to explain osteoblast proliferation behavior upon electrical stimulation. Both for inductively and capacitively coupled stimulation, bone cell proliferation has been enhanced by increased calmodulin levels, whose concentration was promoted by enhanced calcium levels in the cytosol (Aaron et al 2004b). The calcium concentration in the cytosol was due to voltage gated calcium channels for the case of capacitive coupling, and for inductive coupling, it was due to the release of internal calcium stores (Aaron et al 2004b). The increase in osteoblast proliferation in this study might be due to the increased calcium ions in the cytosol, as well. However, cell signaling is very complex, involving various signal transduction pathways which need to be carefully elucidated in the present system.

\section{Conclusions}

To conclude, the optimal voltage window that maximizes osteoblast densities on both conventional and anodized nanotubular titanium was found to be $10 \mathrm{~V}$ to $15 \mathrm{~V}$. Osteoblast density on conventional titanium surfaces was enhanced by $72 \%$ by creating anodized nanotubular surface features and electrically stimulating osteoblasts on them at $15 \mathrm{~V}$. Additionally, osteoblasts responded to lower voltages on anodized compared to conventional titanium. For these reasons, the use of an anodized nanotubular titanium topography 


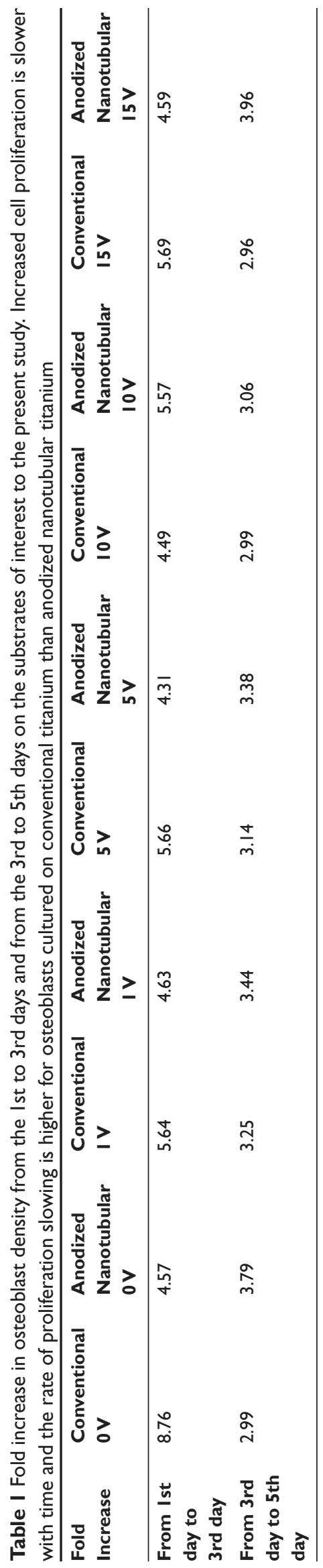

coupled with electrical stimulation has the potential to enhance the performance of titanium for orthopedic implant applications.

\section{Acknowledgments}

The authors would like to thank to the Herman Foundation for funding. The authors report no conflicts of interest in this work.

\section{References}

Aaron RK, Ciombor DM, Simon BJ, et al. 2004a. Treatment of nonunions with electric and electromagnetic fields. Clin Orthoped Relat Res, 419:21-9.

Aaron RK, Boyan BD, Ciombor DM. 2004b. Stimulation of growth factor synthesis by electric and electromagnetic fields. Clin Orthoped, 419:30-7.

Black J. 1987. Electrical stimulation. Its role in growth, repair and remodeling of the musculoskeletal system. New York, NY: Praeger Publishers.

Hartig M, Joos U, Wiesmann HP. 2000. Capacitively coupled electric fields accelerate proliferation of osteoblast-like primary cells and increase bone extracellular matrix formation in vitro. Eur Biophys $J$, 29:499-506.

Karlsson M, Palsgard E, Wilshaw PR, et al. 2003. Initial in vitro interaction of osteoblasts with nano-porous alumina. Biomaterials, 24:3039-46.

Khang D, Park GE, Webster TJ. 2008. Enhanced chondrocyte densities on carbon nanotube composites: The combined role of nano-surface roughness and electrical stimulation. J Biomed Mater Res Part A, 86A:253-60.

Kirson ED, Dbaly V, Tovarys F, et al. 2007. Alternating electric fields arrest cell proliferation in animal tumor models and human brain tumors. Proc Natl Acad Sci U S A, 104:10152-7.

Lee WJ, Alhoshan M, Smyrl WH. 2006. Titanium dioxide nanotube arrays fabricated by anodizing processes. J Electrochem Soc, 153: B499-505.

Mor GK, Varghese OK. 2003. Fabrication of tapered, conical-shaped titania nanotubes. J Mater Res, 18:2588-93.

Nelson FRT, Brighton CT, Ryaby J, et al. 2003. Use of physical forces in bone healing. J Am Acad Orthop Surg, 11:344-54.

Rodriguez R, Kim K, Ong JL. 2002. In vitro osteoblast response to anodized titanium and anodized titanium followed by hydrothermal treatment. J Biomed Mater Res A, 65A:352-8.

Son W, Zhu X, Shin H, et al. 2003. In vivo histological response to anodized and anodized/hydrothermally treated titanium implants. J Biomed Mater Res B Appl Biomater, 66B:520-5.

Supronowicz PR, Ajayan PM, Ullmann KR, et al. 2001. Novel currentconducting composite substrates for exposing osteoblasts to alternating current stimulation. J Biomed Mater Res, 59:499-506.

Yao C, Perla V, McKenzie J, et al. 2005. Anodized Ti and $\mathrm{Ti}_{6} \mathrm{Al}_{4} \mathrm{~V}$ possessing nanometer surface features enhances osteoblast adhesion. J Biomed Nanotechnol, 1:68-73. 
